\title{
PEG10 plays a crucial role in human lung cancer proliferation, progression, prognosis and metastasis
}

\author{
XINZHOU DENG ${ }^{1 *}$, YI HU ${ }^{2 *}$, QIANSHAN DING ${ }^{1}$, RONGFEI HAN ${ }^{1}$, QIAN GUO ${ }^{1}$, JIAN QIN $^{3}$, JIE $^{4}{ }^{4}$, \\ RUIJING XIAO ${ }^{1}$, SUFANG TIAN ${ }^{5}$, WEIDONG HU ${ }^{6}$, QIUPING ZHANG ${ }^{1}$ and JIE XIONG ${ }^{1}$ \\ ${ }^{1}$ Department of Immunology, School of Basic Medical Science, Wuhan University, Wuhan, Hubei 430071; \\ ${ }^{2}$ Department of Clinical Laboratory, The First Affiliated Hosptial of Xiamen University, Xiamen, Fujian 361003; \\ ${ }^{3}$ Central Laboratory, Wuhan University, Renmin Hospital, Wuhan, Hubei 430060; ${ }^{4}$ Central Laboratory, \\ Taihe Hospital, Shiyan, Hubei 442000; Departments of ${ }^{5}$ Pathology and ${ }^{6}$ Oncology, \\ Zhongnan Hospital of Wuhan University, Wuhan, Hubei 430071, P.R. China
}

Received June 24, 2014; Accepted August 14, 2014

DOI: $10.3892 /$ or.2014.3469

\begin{abstract}
Paternally expressed gene 10 (PEG10) has been identified as a genetic imprinted gene, which is important for apoptosis resistance in cancer cells. Mounting evidence suggests that PEG10 is expressed in the majority of hepatocellular carcinoma (HCC) cells with growth-promoting activity. In the present study, we evaluated the correlation between PEG10 expression and the clinicopathological features of lung, breast and HCC tumors, and predicted the relationship between survival and expression levels of PEG10 in lung cancer patients. Furthermore, we chose non-small cell lung cancer cell line A549 as a model to analyze the function of PEG10 in proliferation and metastasis in vitro. Our results revealed that expression of PEG10 was closely correlated with clinical TNM grade and patient prognosis in lung cancer. PEG10 enhanced cell proliferation and promoted tumor cell migration and invasion by upregulating the expression of $\beta$-catenin, MMP- 2 and MMP-9, and decreased the expression of E-cadherin in the A549 cells. Our findings provide significant insight into the molecular mechanisms of lung cancer and offer novel ideas for designing new therapeutic targets for lung carcinoma.
\end{abstract}

\section{Introduction}

Paternally expressed gene 10 (PEG10) is a paternally expressed gene located at human chromosome 7q21 (1). An elevated level of expression has been noted in the majority of human hepatocellular carcinoma cells (HCC), and during the G2/M phase of regenerating mouse liver, although it is absent in normal liver

Correspondence to: Dr Jie Xiong, Department of Immunology, School of Basic Medical Science, Wuhan University, 185 Donghu Road, Wuchang, Wuhan, Hubei 430071, P.R. China

E-mail: annie106xiong@hotmail.com

*Contributed equally

Key words: PEG10, lung cancer, proliferation, metastasis, siRNA cells (1-3). Exogenous expression of PEG10 confers growthpromoting activity and inhibition of its expression retards HCC growth $(4,5)$. PEG10 associates with SIAH1, a mediator of apoptosis, and an imbalance between the expression of PEG10 and SIAH1 may be involved in hepatocarcinogenesis through inhibition of apoptosis (6). Long-term inhibition of PEG10 was found to lead to induction of apoptosis in B-cell chronic lymphocytic leukemia (B-CLL) cells (7). Overexpression of PEG10 may be closely related to the carcinogenesis, progression and prognosis of gallbladder adenocarcinoma (8). PEG10 also plays an important role in placental development $(9,10)$.

Our group has focused on the PEG10 gene in various hematological malignancies for several years. We found that in $\mathrm{CD} 19^{+} \mathrm{CD} 34^{+} \mathrm{B}$ cells from patients with B-cell lineage acute (B-ALL) and chronic lymphocytic leukemia (B-CLL), PEG10 activation by co-stimulation of CXCR5 and CCR7 essentially contributed to resistance to apoptosis (11). In addition, in B-ALL CD $23{ }^{+} \mathrm{CD}^{+} \mathrm{B}$ cells, the interaction of CCL19 and CXCL13 upregulated the expression level of PEG10, and enhanced apoptotic resistance in these cells (12). Our previous study indicated that PEG10 enhanced the invasive capacity and viability of lymphoma cells. We demonstrated that PEG10 promoted the migration of Raji cells by upregulating the expression of matrix metalloproteinase-2 (MMP-2) and MMP-9 (13).

In the present study, we evaluated the correlation between PEG10 expression and the clinicopathological features of lung, breast and HCC tumors using several Gene Expression Omnibus (GEO) datasets with a large number of samples and corresponding clinical information. The results showed that high expression of PEG10 resulted in more rapid tumor growth and spreading in several tumor types, including lung cancer and breast cancer. We postulated that PEG10 may influence the proliferation and metastasis in these cancers. Lung cancer is the leading cause of cancer-related mortality worldwide, with a 5-year survival rate of only $15 \%$. The tumor-node-metastasis (TNM) classification system for lung cancer is a vital guide for determining treatment and prognosis $(14,15)$. We further estimated the relationship between the patient survival and the expression level of PEG10 in lung cancer patients, and compared 
PEG10 expression in lung tumor samples of different T stages. Furthermore, we chose non-small cell lung cancer (NSCLC) A549 cell line as a cell model to analyze the effect of PEG10 on proliferation and metastasis in vitro. Our results revealed that PEG10 plays a crucial role in human lung cancer proliferation and metastasis. PEG10 was closely correlated with lung cancer clinical TNM grade and patient prognosis. PEG10 enhanced cell proliferation and promoted tumor cell migration by upregulating the expression of $\beta$-catenin, MMP-2 and MMP-9, and by decreasing the expression of E-cadherin in the A549 cells. Our findings provide significant insight into the molecular mechanisms of lung cancer and may have clinical relevance for the development of novel targeted therapy.

\section{Materials and methods}

Utilization of publicly available datasets. We downloaded the gene expression profile from the National Center for Biotechnology Information (NCBI) GEO site. We chose several GEO datasets with a large number of samples and corresponding clinical information. GSE30219 and GSE10072 lung cancer datasets were utilized and the clinical information was extracted. GSE3494 was downloaded to analyze the function of PEG10 in breast cancer. GSE14520 was used to explore the role of PEG10 in HCC. The samples pooled in the dataset were divided into two groups according to the expression level of PEG10 in the tumor tissues.

Cell culture and reagents. The A549 cell line was obtained from the Institute of Biochemistry and Cell Biology, Shanghai Institute for Biological Science, Chinese Academy of Sciences. Cells were maintained in RPMI-1640 containing $100 \mathrm{U} / \mathrm{ml}$ penicillin, $100 \mathrm{mg} / \mathrm{ml}$ streptomycin and $10 \%$ fetal bovine serum (FBS) at $37^{\circ} \mathrm{C}$ in a humidified incubator supplemented with $5 \% \mathrm{CO}_{2}$ in air. Cell morphology was observed by light microscopy. The primary antibodies used in the present study included mouse polyclonal antibody to human PEG10 (Abcam, Cambridge, UK), mouse monoclonal antibody to $\beta$-catenin (Santa Cruz Biotechnology, Santa Cruz, CA, USA), mouse monoclonal antibody to $\beta$-actin (Abmart, Shanghai, China), rabbit polyclonal antibody to E-cadherin (Biosynthesis Biotechnology, Beijing, China) and monoclonal antibodies to MMP-2/-9 (Epitomics, Burlingame, CA, USA). Other reagents included Cell Counting Kit-8 (CCK-8; Dojindo), Oligofectamine, Opti-MEM and primers for PEG10, $\beta$-catenin, E-cadherin, MMP-9/-2, C-myc and GAPDH (Invitrogen).

siRNA and transfection. PEG10 siRNA and the scramble siRNA were designed and synthesized as previously reported (13). Cells were grown to $\sim 60 \%$ confluency in 6-well plates (NEST Biotechnology Co., Ltd., Wuxi, China) one day before transfection. According to the manufacturer's instructions, we diluted the siRNAs with the appropriate concentration in Opti-MEM I to form complexes. A549 cells were transiently transfected with the complexes obtained by Oligofectamine. Cells were cultured with serum-free medium supplemented with $10 \%$ FBS 6 -h post transfection, and then incubated at $37^{\circ} \mathrm{C}$ in air containing $5 \% \mathrm{CO}_{2}$ for $48-72 \mathrm{~h}$. The viability of the cells was tested by trypan blue staining with a hemocytometer.
Cell proliferation assay. A549 cells were dispensed in 96-well plates at 2,000/well and allowed to adhere overnight. After transfection with $100 \mathrm{nM}$ PEG10 siRNA or scramble siRNA for $48 \mathrm{~h}, \mathrm{CCK}-8$ solution $(10 \mu \mathrm{l})$ was added to each well of the plate at different culture points and incubated for another $3 \mathrm{~h}$. The absorbance was measured using a PerkinElmer 2030 VICTOR X Multilabel Plate Reader (Perkin-Elmer, Waltham, MA, USA) at $450 \mathrm{~nm}$.

Clonogenic survival assay. After transfection with $100 \mathrm{nM}$ PEG10 siRNA or scramble siRNA for $48 \mathrm{~h}$, A549 cells were seeded at 500 cells/well in 6-well plates. Plates were incubated in a $37^{\circ} \mathrm{C}, 5 \% \mathrm{CO}_{2}$ incubator for 2 weeks. Medium was replaced every $72 \mathrm{~h}$. When colonies became visible ( 2 weeks), the cells were fixed and stained with $0.1 \%$ crystal violet (Sigma-Aldrich), then photographed and counted under a microscopic field at a magnification of x10. Each assay was performed in triplicate on four independent occasions.

Wound healing assay. Migration ability was assessed by a wound healing assay as previously described (16). A549 cells were seeded in triplicate wells of 24-well plates and incubated in RPMI-1640 containing 10\% FBS until they reached subconfluency. A vertical wound was created in the cell monolayer in each well using a sterile P10 micropipette tip after transfection with $100 \mathrm{nM}$ PEG10 siRNA or scramble siRNA for $48 \mathrm{~h}$. The wells were washed twice with phosphate-buffered saline (PBS), then removed and replaced by $1 \mathrm{ml}$ of serum-free culture medium. Images of wound edges for each condition were captured at time 0, 6, 12 and $24 \mathrm{~h}$ using a Canon 600D camera (Japan). All analyses were repeated in triplicate.

Matrigel invasion assay. The Matrigel Transwell assay was performed as previously described $(13,17)$. A549 cells were pretreated with $100 \mathrm{nM}$ PEG10 siRNA or scramble siRNA for $48 \mathrm{~h}$, then digested, centrifuged and rendered into a singlecell suspension in RPMI-1640 with $0.1 \%$ BSA. Cells $\left(5 \times 10^{4}\right)$ were added into the upper chambers pre-coated with Matrigel, which were separated from the lower wells by a 6.5-mm-diameter polycarbonate membrane with an $8-\mu \mathrm{m}$ pore size filter. The lower chambers were filled with $600 \mu \mathrm{l}$ of RPMI-1640 containing $10 \% \mathrm{FBS}$. After incubation at $37^{\circ} \mathrm{C}$ in $5 \% \mathrm{CO}_{2}$ for $24 \mathrm{~h}$, the medium was discarded. The cells that remained in the upper chamber were removed with cotton swabs and washed with PBS. The membrane was fixed in methanol and stained with $0.1 \%$ crystal violet. Cells which migrated into the bottom surface of the filter were counted with ImageJ programme (National Institutes of Health, USA) by a light microscope. The results were normalized to the control groups and repeated five times.

$R N A$ extraction and real-time quantitative $R T-P C R$. Total RNA was isolated by TRIzol (Invitrogen, Carlsbad, CA, USA) and quantified by NanoDrop 2000 (Thermo Scientific, Waltham, MA, USA). Total RNA (1 $\mu \mathrm{g})$ from each sample was reverse transcribed with random primers using the Promega reverse transcriptase kit (USA) according to the protocol of the manufacturer. Real-time quantitative RT-PCR (qPCR) analyses were performed using SYBR Premix Ex Taq $^{\mathrm{TM}}$ based 
detection (Takara, Japan) with the $\mathrm{iQ}^{\mathrm{TM}} 5$ instrument (Bio-Rad, USA). The sequences of the specific primers were as follows: PEG10 forward, 5'-AACAACAACAACAACTCCAAGC-3' and reverse, 5'-TCTGCACCTGGCTCTGCAG-3'; $\beta$-catenin forward, 5'-TGGTGCCCAGGGAGAACCCC-3' and reverse, 5'-TGTCACCTGGAGGCAGCCCA-3'; E-cadherin forward, 5'-ATTTTTCCCTCGACACCCGAT-3' and reverse, 5'-TCCCA GGCGTAGACCAAGA-3'; C-myc forward, 5'-AATAGAGCT GCTTCGCCTAGA-3' and reverse, 5'-GAGGTGGTTCATAC TGAGCAAG-3'; MMP-2 forward, 5'-TGACATCAAGGGCA TTTCAGGAGC-3' and reverse, 5'-GTCCGCCAAATGAACC GGTCCTTG-3'; MMP-9 forward; 5'-GAGGTTCGACGTGA AGGCGCAGATG-3' and reverse, 5'-CATAGGTCACGTAGC CCACTTGGTC-3'; Twist forward, 5'-GGGAGTCCGCAGTC TTAC-3' and reverse, 5'-CCTGTCTCGCTTTCTCTTT-3'; Vimentin forward, 5'-GCCCTAGACGAACTGGGTC-3' and reverse, 5'-GGCTGCAACTGCCTAATGAG-3'; p53 forward, 5'-GGGGAGCAGGGCTCACTCCA-3' and reverse, 5'-GTG GAGCCCCGGGACAAAGC-3'; p21 forward, 5'-TCCTGGA GCAGACCACCCCG-3' and reverse, 5'-GGGGTGGGACAG GCACCTCA-3'; Snail forward, 5'-TCGGAAGCCTAACTA CAGCGA-3' and reverse, 5'-AGATGAGCATTGGCAGCG AG-3'; GAPDH forward, 5'-CTGGGCTACACTGAGCA CC-3' and reverse, 5'-AAGTGGTCGTTGAGGGCAATG-3'. The expression level of relative mRNA was normalized to human GAPDH and calculated using the $2^{-\Delta \Delta \mathrm{Ct}}$ method.

Western blotting. Cells were washed with PBS and lysed with RIPA (50 mM Tris- $\mathrm{HCl} \mathrm{pH} 7.6,150 \mathrm{mM} \mathrm{NaCl}, 2 \mathrm{mM}$ EDTA, 2 mM EGTA, 0.1\% Triton-X) containing protease inhibitor PMSF. The total protein concentration was measured using the BCA protein assay kit (Thermo Scientific) on the PerkinElmer 2030 VICTOR X Multilabel Plate Reader. Whole cell lysates were boiled at $100^{\circ} \mathrm{C}$ for $5 \mathrm{~min}$ in equal volumes of loading buffer $(0.5 \mathrm{M}$ Tris- $\mathrm{HCl} \mathrm{pH} 6.8,2 \% \mathrm{SDS}, 0.05 \%$ bromphenol-blue, $20 \%$ 2-mercaptoethanol and $10 \%$ glycerol). All samples were subjected to $10 \%$ SDS-PAGE, separated by electrophoresis and transferred to PVDF membranes. After blocking for $2 \mathrm{~h}$ in TBST containing 5\% non-fat milk, the membranes were incubated with the primary antibody diluted in TBST containing 5\% non-fat milk with gentle shaking at $4^{\circ} \mathrm{C}$ overnight. After washing four times, the membranes were incubated with the HRP-conjugated secondary antibodies for $2 \mathrm{~h}$ at room temperature. The signals were detected using an enhanced chemiluminescence detection kit (Thermo Scientific, Rockford, IL, USA).

Statistical analysis. Statistical analysis was conducted using GraphPad Prism 5.0. Values are shown as means \pm SD of at least of three independently conducted experiments. Statistical significance was assessed by t-test followed by paired comparisons. Kaplan-Meier plots were constructed, and a log-rank test was used to evaluate differences in PEG10 expression levels for overall survival. $\chi^{2}$ test was used to analyze the correlation between PEG10 expression and the clinicopathologic features. The difference in PEG10 expression in tumors of different $\mathrm{T}$ stage was determined by analysis of variance (ANOVA). $\mathrm{P}<0.05$ was considered to indicate a statistically significant result.
Table I. Correlation between PEG10 expression and the clinicopathological features of the lung cancer cases from GSE30219.

\begin{tabular}{lrrrrr}
\hline & \multicolumn{5}{c}{$\begin{array}{c}\text { PEG10 } \\
\text { expression }\end{array}$} \\
\cline { 3 - 4 } Characteristics & $\begin{array}{c}\text { No. of } \\
\text { patients }\end{array}$ & High & Low & $\begin{array}{c}\chi^{2} \\
\text { value }\end{array}$ & P-value \\
\hline Age (years) & & & & & $0.0010^{\mathrm{b}}$ \\
$\leq 55$ & 82 & 31 & 51 & 10.76 & \\
$>55$ & 211 & 41 & 170 & & \\
Gender & & & & & 0.1247 \\
Male & 252 & 58 & 194 & 2.36 & \\
Female & 41 & 14 & 27 & & \\
T stage & & & & & $0.0204^{\mathrm{a}}$ \\
1 & 167 & 30 & 137 & 5.37 & \\
$2-4$ & 118 & 35 & 83 & & \\
N stage & & & & & $<0.0001^{\mathrm{b}}$ \\
Positive & 92 & 36 & 56 & 16.88 & \\
Negative & 195 & 33 & 162 & & \\
Metastasis & & & & & $0.1910^{\mathrm{c}}$ \\
Yes & 8 & 4 & 4 & 1.71 & \\
No & 281 & 66 & 215 & & \\
\hline
\end{tabular}

The top $25 \%$ of samples with the highest PEG10 expression was considered as the PEG10 high-expression group; the remaining $75 \%$ of the samples was considered as the low-expression group ( $\chi^{2}$ text was used). ${ }^{\mathrm{a}} \mathrm{P}<0.05,{ }^{\mathrm{b}} \mathrm{P}<0.01$. ${ }^{\mathrm{c}}$ Yates correction.

\section{Results}

PEG10 is closely correlated to clinicopathological features of lung cancer. We chose several GEO datasets with a large number of samples and corresponding clinical information. The samples pooled in the lung cancer dataset GSE30219 were divided into two groups according to the expression level of PEG10 in tumor tissues and $\chi^{2}$ test was used. The top $25 \%$ samples with the highest PEG10 expression were considered as the high-expression group; and the remaining $75 \%$ of the samples as the low-expression group. As shown in Table I, PEG10 expression was associated with age $(\mathrm{P}=0.0010)$, $\mathrm{T}$ stage $(\mathrm{P}=0.0204)$ and $\mathrm{N}$ stage $(\mathrm{P}<0.0001)$ in the lung cancer cases. Even though no significant difference was found, there was an obvious trend that PEG10 was expressed more frequently in the tumor tissues of patients with distant metastasis. These results imply that PEG10 is associated with lung cancer proliferation and metastasis.

Similarly, GSE3494 was downloaded to analyze the function of PEG10 in breast cancer. As shown in Table II, PEG10 expression was significantly correlated with age $(\mathrm{P}=0.0495)$, p53 status $(\mathrm{P}=0.0102)$, p53 mutation $(\mathrm{P}=0.0014)$, Elston histologic grade $(\mathrm{P}=0.0001)$, tumor size $(\mathrm{P}=0.0031)$ and lymph node metastasis $(\mathrm{P}=0.0149)$ in the breast cancer cases. GSE14520 was also used to explore the role of PEG10 in HCC, yet both the results of the retrospective and prospective analyses were not significant (data not shown). 
Table II. Correlation between PEG10 expression and the clinicopathological features of the breast cancer cases from GSE3494.

\begin{tabular}{|c|c|c|c|c|c|}
\hline \multirow[b]{2}{*}{ Characteristics } & \multirow{2}{*}{$\begin{array}{c}\text { No. of } \\
\text { patients }\end{array}$} & \multicolumn{2}{|c|}{$\begin{array}{l}\text { PEG10 } \\
\text { expression }\end{array}$} & \multirow{2}{*}{$\begin{array}{c}\chi^{2} \\
\text { value }\end{array}$} & \multirow[b]{2}{*}{ P-value } \\
\hline & & High & Low & & \\
\hline Age (years) & & & & & $0.0495^{\mathrm{a}}$ \\
\hline$<55$ & 75 & 25 & 50 & 3.86 & \\
\hline$\geq 55$ & 176 & 38 & 138 & & \\
\hline p53 status & & & & & $0.0102^{\mathrm{b}}$ \\
\hline Positive & 58 & 22 & 36 & 6.61 & \\
\hline Negative & 193 & 41 & 152 & & \\
\hline p53 mutation & & & & & $0.0014^{\mathrm{b}}$ \\
\hline Yes & 72 & 28 & 44 & 10.21 & \\
\hline No & 179 & 35 & 144 & & \\
\hline ER status & & & & & 0.5737 \\
\hline Positive & 213 & 53 & 160 & 0.32 & \\
\hline Negative & 34 & 10 & 24 & & \\
\hline PR status & & & & & 0.5664 \\
\hline Positive & 61 & 44 & 17 & 0.33 & \\
\hline Negative & 190 & 144 & 46 & & \\
\hline $\begin{array}{l}\text { Elston histological } \\
\text { grade }\end{array}$ & & & & & $0.0001^{\mathrm{b}}$ \\
\hline 1 & 67 & 6 & 61 & 13.90 & \\
\hline 2 & 128 & 35 & 93 & & \\
\hline 3 & 54 & 20 & 34 & & \\
\hline Tumor size & & & & & $0.0031^{\mathrm{b}}$ \\
\hline$\geq 2 \mathrm{~cm}$ & 139 & 45 & 94 & 8.77 & \\
\hline$<2 \mathrm{~cm}$ & 112 & 18 & 94 & & \\
\hline $\begin{array}{l}\text { Lymph node } \\
\text { metastasis }\end{array}$ & & & & & $0.0149^{\mathrm{a}}$ \\
\hline Yes & 84 & 29 & 55 & 5.92 & \\
\hline No & 158 & 32 & 126 & & \\
\hline
\end{tabular}

The top 25\% of samples with the highest PEG10 expression was considered as the PEG10 high-expression group; the remaining $75 \%$ of the samples was considered as the low-expression group ( $\chi^{2}$ text was used). ${ }^{\mathrm{a}} \mathrm{P}<0.05,{ }^{\mathrm{b}} \mathrm{P}<0.01$.

We applied GSE30219 dataset to further analysis the survival curves for lung cancer patients. The top 50\% samples with the highest PEG10 expression were counted as the highexpression group, and the remaining $50 \%$ of the samples as the low-expression group. The Kaplan-Meier method and log-rank test were used to compare the survival of patients with lung adenocarcinoma (Fig. 1A) and basaloid carcinoma (Fig. 1B) in the high-expression and low-expression subgroups. The data indicated that there was a significant difference between the high PEG10 expression group and the low-expression group $(\mathrm{P}<0.05)$ in terms of patient survival. Fig. $1 \mathrm{C}$ shows the relationship between expression levels of PEG10 and lung cancer samples in different $\mathrm{T}$ stages $(\mathrm{P}<0.05)$, but not in a
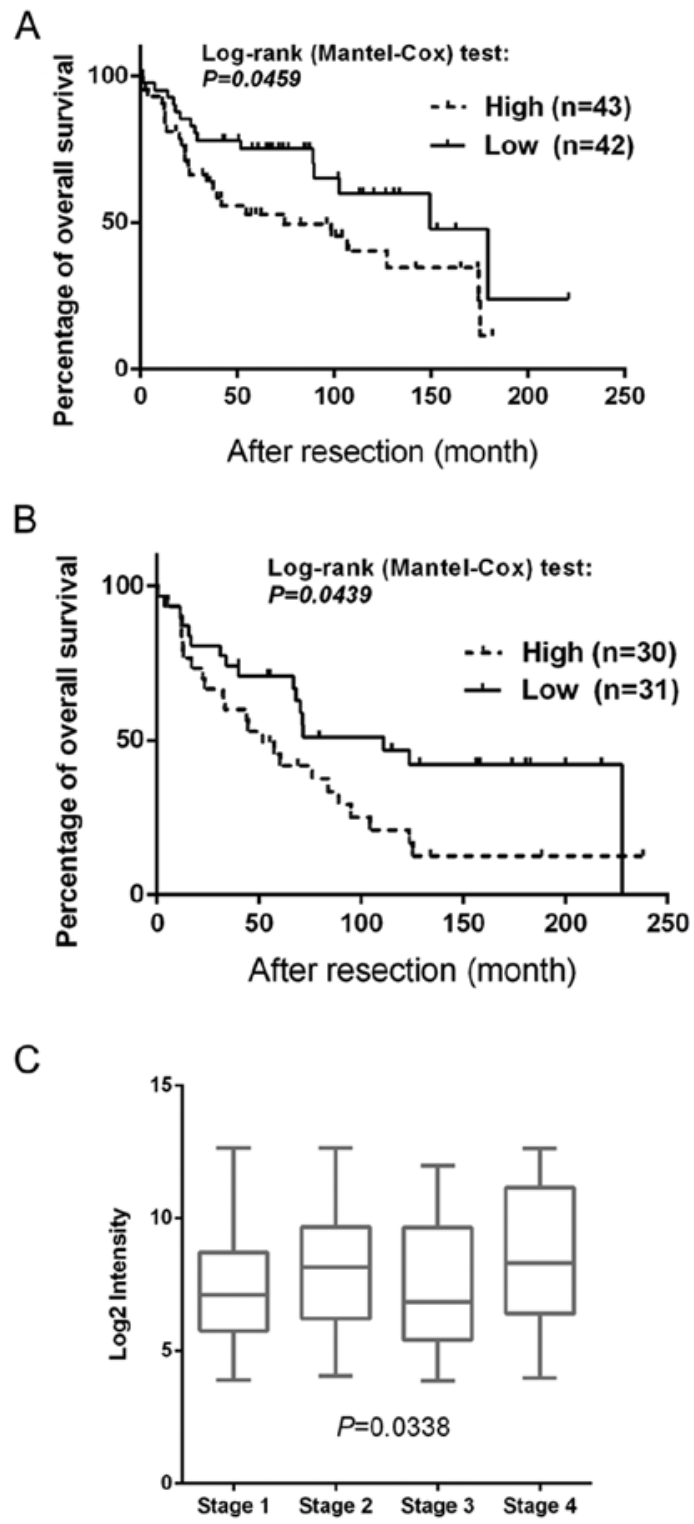

Expression of PEG10 in tumor samples of different T stages

D

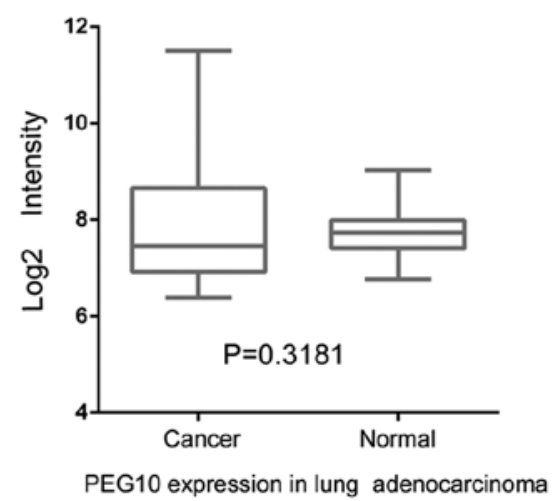

Figure 1. Lung cancer dataset analysis. The Kaplan-Meier method was used to estimate survival curves for lung cancer patients in GSE30219. Log-rank test was used to compare the differences between curves. The top 50\% samples with the highest expression were considered as the high-expression group, and the remaining $50 \%$ of the samples as the low-expression group. (A) Lung adenocarcinoma $(\mathrm{P}<0.05)$ and $(\mathrm{B})$ basaloid carcinoma $(\mathrm{P}<0.05)$. (C) The relationship between expression levels of PEG10 and lung cancer samples of different $\mathrm{T}$ stages $(\mathrm{P}<0.05)$. (D) Expression levels in cancer and normal tissues in the lung adenocarcinoma dataset GSE10072 (paired samples, $\mathrm{n}=33, \mathrm{P}=0.3181$ ). $\mathrm{PEG} 10$, paternally expressed gene 10 . 


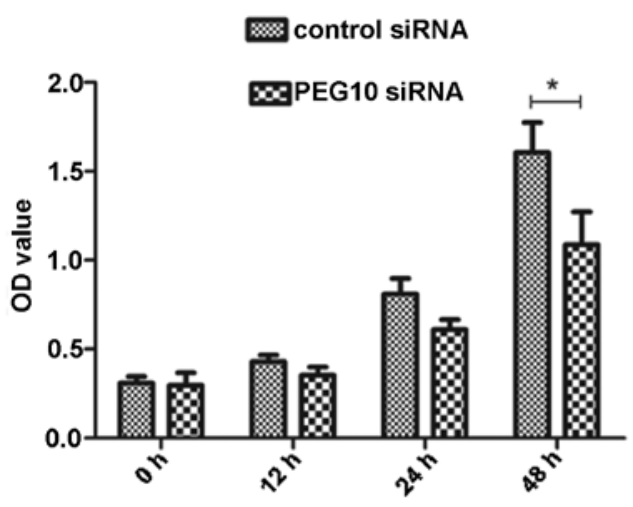

Figure 2. Effect of PEG10 on the proliferation of A549 cells. Proliferation was determined by CCK- 8 assay at the indicated times after A549 cells were transfected with control siRNA or PEG10 siRNA for $48 \mathrm{~h}$. Data are expressed as the mean value $\pm \mathrm{SD}$ of three experiments in each group $\left({ }^{*} \mathrm{P}<0.05\right)$. PEG10, paternally expressed gene 10 .

stage-dependent level. High expression of PEG10 may be a poor prognostic marker in lung cancer. We also compared the expression levels of PEG10 in cancer tissues and normal tissues in lung adenocarcinoma dataset GSE10072 (paired samples, $n=33)$. The results showed that there was no statistical difference (Fig. 1D).

PEG10 promotes $A 549$ proliferation. We firstly measured the interference rate of PEG10 siRNA. A549 cells were transfected with 100 nM PEG10 siRNA or scramble siRNA. We collected the cells for mRNA and protein extraction, respectively. The results showed that PEG10 was inhibited in a dose-dependent manner, and the lowest expression at the mRNA level was at $48 \mathrm{~h}$ and at the protein level was $72 \mathrm{~h}$ (data not shown). To assess the effect of proliferation by PEG10, A549 cells were transfected with 100 nM PEG10 siRNA or scramble siRNA for $48 \mathrm{~h}$ and then seeded in culture plates. Cell viability was measured by CCK- 8 solution, while colony formation ability was assessed by clonogenic survival assay. We found that inhibition of PEG10 reduced the proliferation rate of A549 cells compared with the control group in a time-dependent manner, particularly at $48 \mathrm{~h}(\mathrm{P}<0.05)$ (Fig. 2).

PEG10 enhances the carcinogenesis of A549 cells. After incubation in a $37^{\circ} \mathrm{C}, 5 \% \mathrm{CO}_{2}$ incubator for 2 weeks, colonies were fixed and stained, and the numbers were counted. A smaller number of colonies was observed in the siRNA PEG10transfected group compared with the number in the control group ( $\mathrm{P}<0.05)$ (Fig. 3), suggesting that PEG10 significantly promotes A549 cell growth.

PEG10 promotes A549 cell migration and invasion. To investigate the migration and invasion abilities of PEG10 in lung cancer, we performed a wound healing assay (Fig. 4) and Matrigel Transwell assay (Fig. 5) in A549 cells transfected with PEG10 siRNA or not transfected. The cell migration rate was significantly reduced after suppression of PEG10 by RNA interference as detected in the wound healing assay. During the $48 \mathrm{~h}$ time course, cells in the PEG10 inhibition group spread along the wound edges more slowly when compared with the control cells in a time-dependent manner. We also found
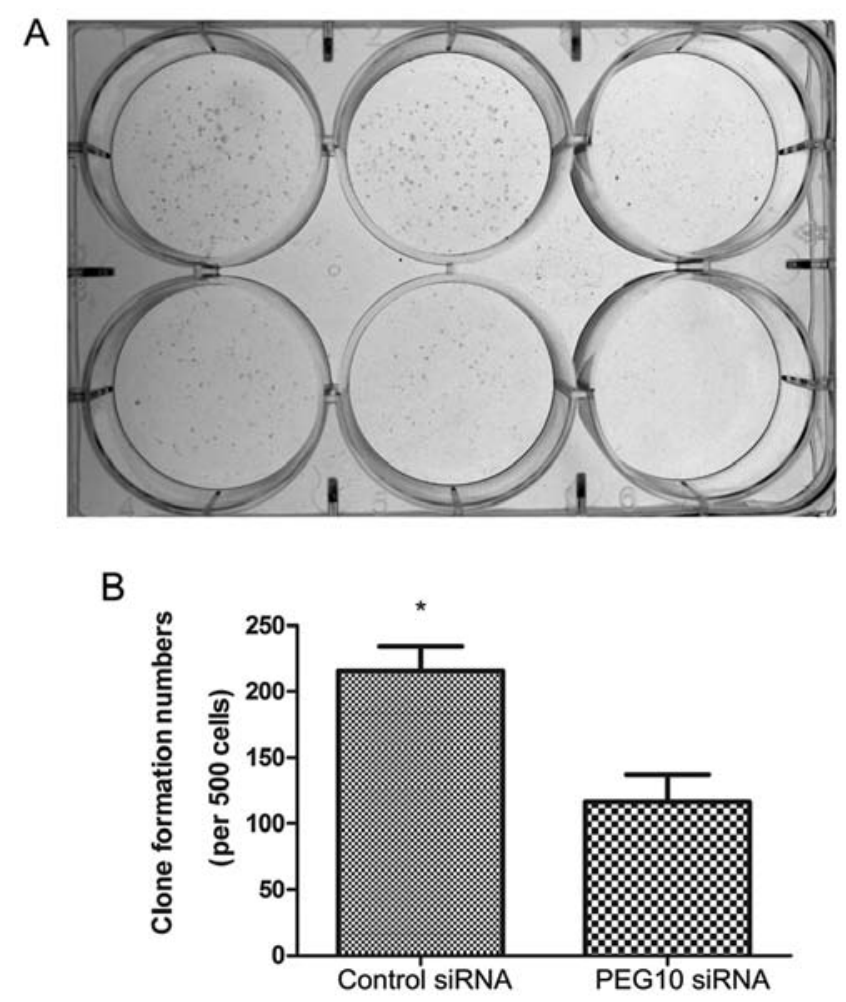

Figure 3. Effect of PEG10 as determined by a clonogenic survival assay. (A) Prior to the colony formation experiment, A549 cells were transiently transfected with control or PEG10 siRNA for $48 \mathrm{~h}$, and the cells were then seeded at 500 cells/well in 6 -well plates. Plates were incubated in a $37^{\circ} \mathrm{C}, 5 \%$ $\mathrm{CO}_{2}$ incubator for another 2 weeks. (B) The numbers of colonies formed in $\mathrm{A}$ were counted $\left({ }^{* *} \mathrm{P}<0.01\right)$. PEG10, paternally expressed gene 10 .

that PEG10 siRNA significantly reduced the cell invasion through the micropore membranes into the lower chambers as compared with the control group. These data provide strong evidence that PEG10 enhances the metastatic ability of A549 cells.

PEG10 affects the expression of several migration-related molecules. The canonical Wnt/ $\beta$-catenin signaling pathway has been well characterized in promoting lung cancer cell migration (18-20). Endogenous $\beta$-catenin interacts with E-cadherin in the membrane to mediate cell adhesion in many types of cancers. As a member of the cadherin superfamily, E-cadherin is involved in maintaining cell polarity and organizing the epithelium by strengthening intercellular adhesion; loss of E-cadherin, a hallmark of epithelial-mesenchymal transition (EMT), can significantly affect metastatic dissemination (21-24). In the present study, A549 cells were pretreated with $100 \mathrm{nM}$ PEG10 siRNA or scramble siRNA for 48 or $72 \mathrm{~h}$. Then we collected the cells for mRNA and protein extraction, respectively. Here, we demonstrated that PEG10 is associated with expression of $\beta$-catenin and E-cadherin. Silencing of PEG10 significantly reduced the expression of $\beta$-catenin and enhanced the expression of E-cadherin in the A549 cells (Fig. 6). Yet, the mRNA levels of mesenchymal transcription factor Twist and Slug were not appreciably different. Among the proteinases capable of degrading the extracellular matrix (ECM), MMP-2 and MMP-9 appear to be particularly 

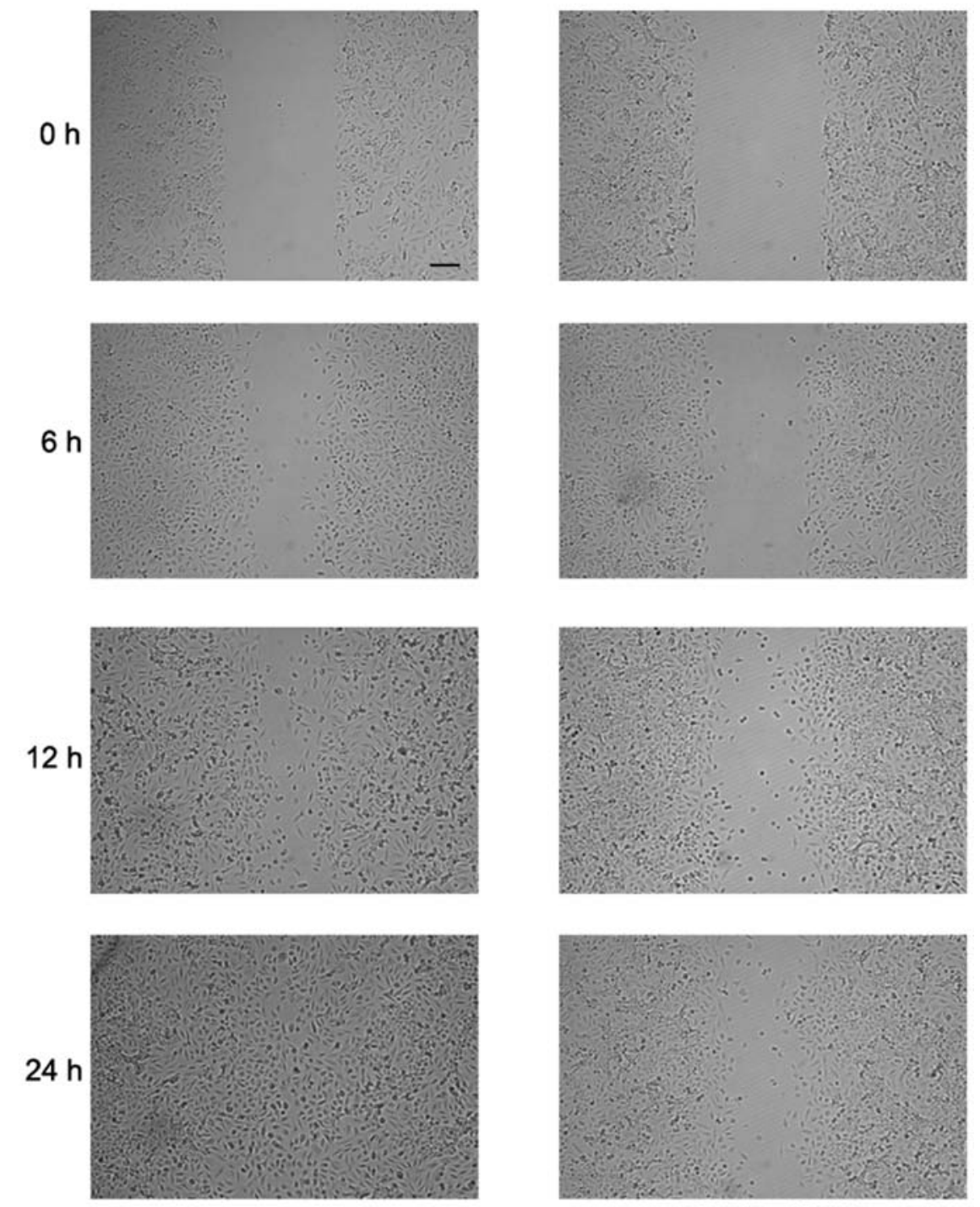

Control siRNA

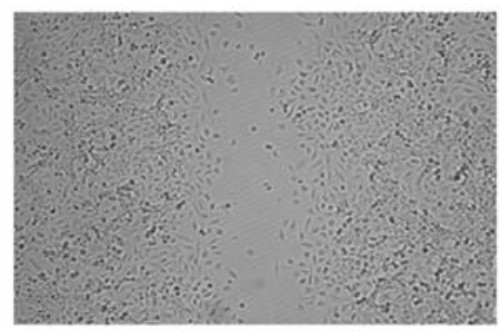

\section{PEG10 siRNA}

Figure 4. PEG10 enhances A549 cell migration. Cell migration was determined by a wound healing assay at different time point in A549 cells after incubation in the absence or presence of $100 \mathrm{nM}$ PEG10 siRNA for $48 \mathrm{~h}$. Scale bar, $200 \mu \mathrm{m}$. PEG10, paternally expressed gene 10.

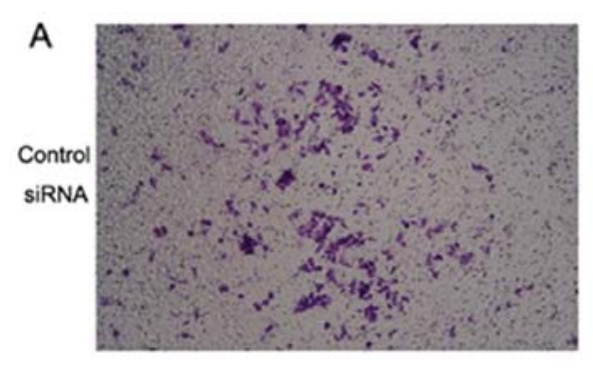

B
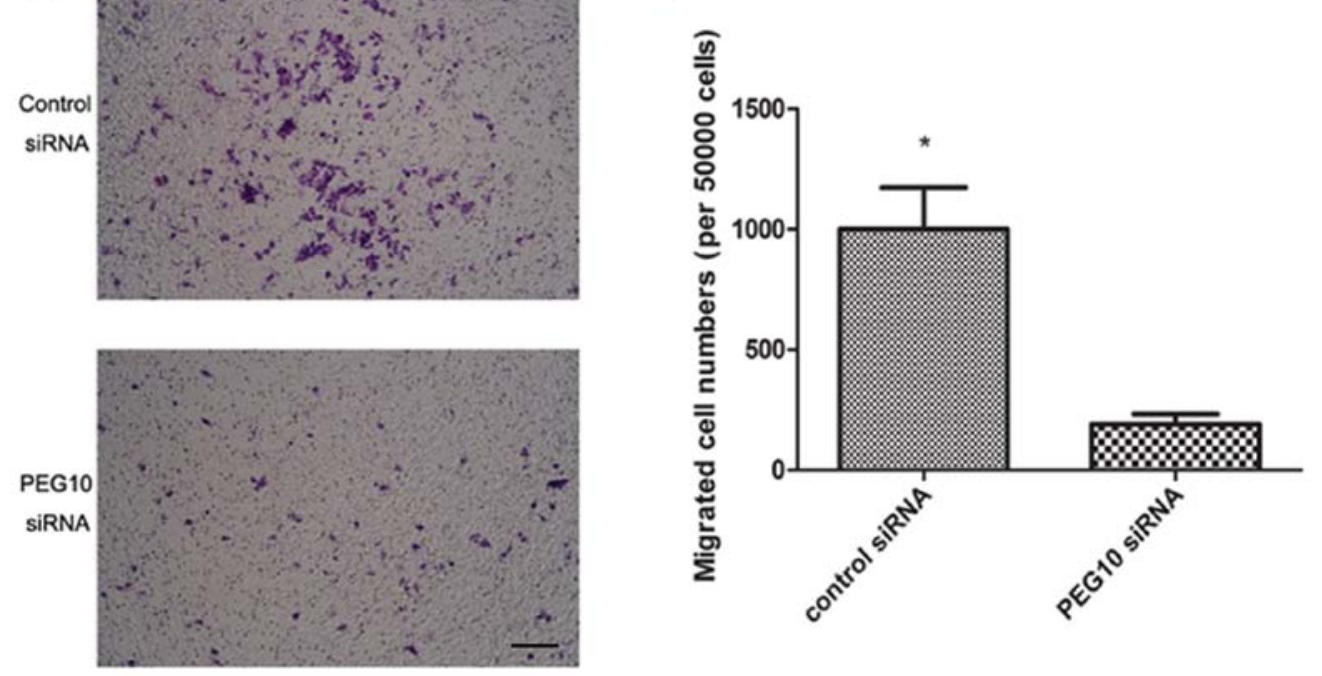

Figure 5. PEG10 enhances A549 cell invasion. (A) Cell invasion was assessed by Matrigel Transwell assay in A549 cells after the cells were treated with $100 \mathrm{nM}$ PEG10 siRNA for $48 \mathrm{~h}$. The cells which migrated into the bottom surface of the filter were stained. (B) The number of invasive cells in A were determined. Data are presented as means \pm SD of three independent determinations ( $\left.{ }^{*} \mathrm{P}<0.05\right)$. Scale bar, $200 \mu \mathrm{m}$. PEG10, paternally expressed gene 10. 

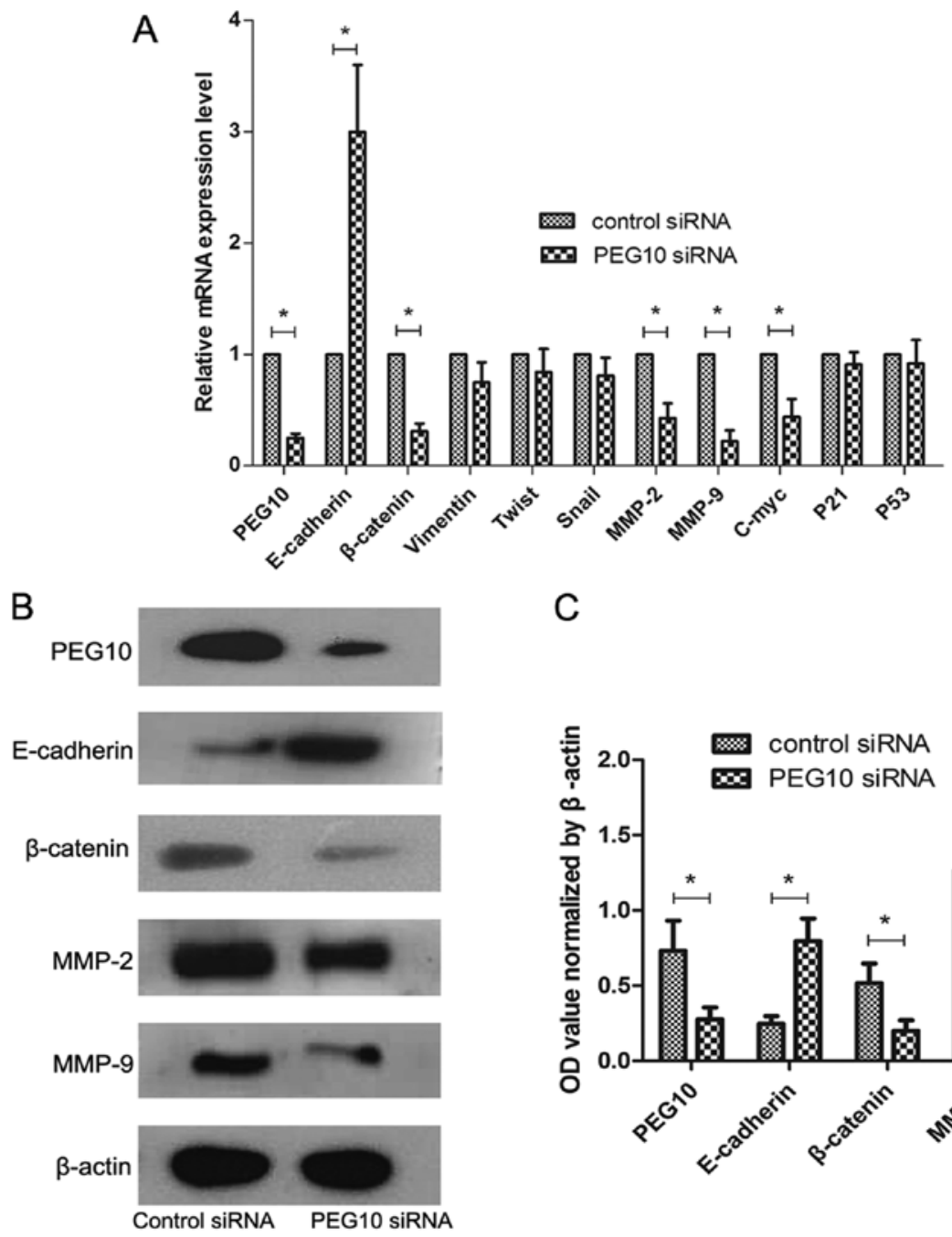

C

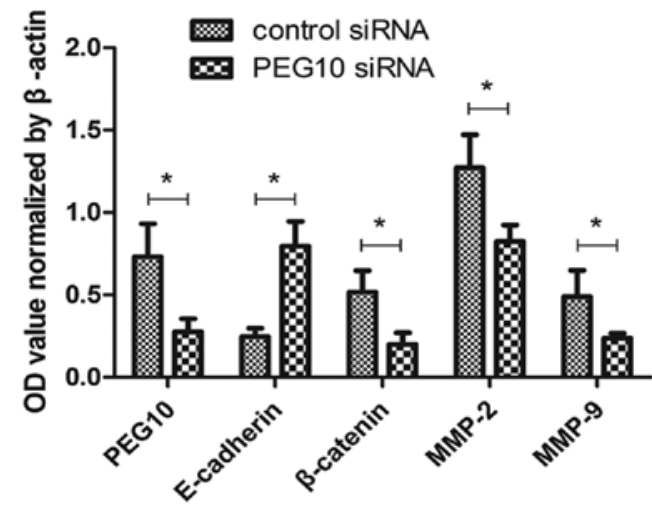

Figure 6. PEG10 affects the expression of several migration-related molecules. The expression levels of mRNA (A) and protein (B and C) of PEG10, $\beta$-catenin, E-cadherin, MMP-2 and MMP-9 and other molecules were analyzed after transfection with PEG10 siRNA or control siRNA, respectively. Bars represent the relative mRNA copies or optical density (OD) values normalized to actin $\left({ }^{*} \mathrm{P}<0.05,{ }^{* *} \mathrm{P}<0.01\right)$. Data from real-time RT-PCR (A) and western blotting $(\mathrm{C})$ were expressed as means value \pm SD from at least three independent experiments. PEG10, paternally expressed gene 10.

important $(25,26)$. Our results also revealed that MMP-2 and MMP-9 mRNA and protein expression levels declined when PEG10 expression was effectively suppressed (Fig. 6). In conclusion, we speculate that PEG10 is associated with invasion of A549 cells by affecting the expression of $\beta$-catenin, E-cadherin, MMP-2 and MMP-9.

\section{Discussion}

Lung cancer is a malignant disease with the highest rate in terms of incidence and mortality. The lung is the most common cancer site in males, and lung cancer comprising $17 \%$ of all newly diagnosed cancer cases and $23 \%$ of the total cancerrelated deaths (27). An estimate from the European Union implies that lung cancer will likely overtake breast cancer and become the leading cause of cancer-related mortality in women (28). Early diagnosis and timely monitoring of tumor recurrence and metastasis are extremely important for improving the 5-year survival rate of lung cancer patients. At the time of diagnosis, the majority of patients with NSCLC already suffer from advanced or metastatic disease, which is associated with poor prognosis $(29,30)$. In recent years, identification and characterization of specific tumor-associated genes has accelerated the development of molecule-targeting drugs in lung cancer.

PEG10 has been identified as a maternally imprinted and paternally expressed gene that functions as a transcriptional factor $(1,4,6)$. Mounting evidence suggests that PEG10 is highly expressed in the majority of $\mathrm{HCC}$ with a growth-promoting activity $(2,3)$. Our previous study confirmed that PEG10 functions as an important oncogene by promoting tumor growth and increasing tolerance to apoptosis. We demonstrated that PEG10 enhanced the invasive capacity by upregulating expression of MMPs (11-13). In the present study, our results revealed that PEG10 expression was closely associated with the TNM classification in lung cancer. Furthermore, we investigated the relationship between patient survival and different expression levels of PEG10 in lung cancer patients. Our results suggest that high expression of PEG10 may be a critical indicator for a poor prognosis in lung cancer. 
In order to better understand the role of PEG10 in lung cancer, we applied in vitro experiments to confirm the results from the bio-information analysis with clinical data. Here, we demonstrated that PEG10 significantly enhanced proliferation (Fig. 2) and clone formation (Fig. 3) of A549 cells. Moreover, the migration and invasion abilities were both significantly reduced when PEG10 expression was suppressed. These results strongly imply that PEG10 is associated with lung cancer cell proliferation, carcinogenesis and migration.

The Wnt/ $\beta$-catenin pathway is one of the most important signal transduction pathways in tumorigenesis and progression. $\beta$-catenin is a $92-\mathrm{kDa}$ protein that together with E-cadherin plays a role in cell-cell adhesion and is involved in intracellular signaling (31-33). E-cadherin is involved in maintaining cell polarity and organizing the epithelium by strengthening intercellular adhesion. The extracellular regions of cadherins on opposing cells bind to one another, and their cytoplasmic regions bind to $\beta$-catenin. Reduction in E-cadherin is often connected with an increased free cytoplasmic level of $\beta$-catenin, which could translate into increased $\beta$-catenin transcription if it escapes cytoplasmic degradation. Several studies suggest that $\beta$-catenin-mediated transcription induces Slug or Twist gene expression, which further suppresses E-cadherin and contributes to EMT (33-35). Our data showed that downregulation of PEG10 led to a decrease in the expression level of $\beta$-catenin in A549 cells, while the expression level of E-cadherin was markedly upregulated (Fig. 6). We postulate that $\beta$-catenin and E-cadherin may be important downstream molecules of PEG10 in tumor metastasis.

Tumor cell invasion is a multistep process, in which extensive degradation of the matrix is the most critical step. Hallmarks of tumor cell invasion and migration include the excessive degradation of the matrix. MMP-2 and MMP-9 are enzymes that degrade collagen type IV, which are thought to be associated with the degradation of ECM structures of tissue and blood vessels and migration to other organs (36-38). In the present study, the expression levels of MMP-2 and MMP-9 were significantly inhibited at both the mRNA and protein levels, after knockdown of PEG10 expression by PEG10 siRNA (Fig. 6). MMP-2 and MMP-9 are closely related to the invasive phenotype of cancer cells. Our results further suggest that PEG10 promotes the invasion of lung cancer, which in accordance with the results of the Matrigel Transwell assay, lead to the conclusion that MMP-2 and MMP-9 are downstream molecules of PEG10 for promoting A549 metastasis.

In conclusion, the present study demonstrated that PEG10 significantly promoted proliferation, migration and invasion of A549 cells. PEG10 is closely correlated with clinical grade, and it may be regarded as a potential prognostic marker for the aggressiveness of lung cancer. Our results add to the understanding of the role of PEG10 and elucidate the possible clinical relevance for the development of a novel target therapeutic agent.

\section{Acknowledgements}

The present study was supported by the National Natural Science Foundation of China (nos. 81270607, 30971280 and 30801336).

\section{References}

1. Ono R, Kobayashi S, Wagatsuma $\mathrm{H}$, et al: A retrotransposonderived gene, $P E G 10$, is a novel imprinted gene located on human chromosome 7q21. Genomics 73: 232-237, 2001.

2. Li CM, Margolin AA, Salas M, et al: PEG10 is a c-MYC target gene in cancer cells. Cancer Res 66: 665-672, 2006.

3. Tsou AP, Chuang YC, Su JY, et al: Overexpression of a novel imprinted gene, PEG10, in human hepatocellular carcinoma and in regenerating mouse livers. J Biomed Sci 10: 625-635, 2003.

4. Tsuji K, Yasui K, Gen Y, et al: PEG10 is a probable target for the amplification at 7q21 detected in hepatocellular carcinoma. Cancer Genet Cytogenet 198: 118-125, 2010.

5. Lux H, Flammann H, Hafner $M$ and Lux A: Genetic and molecular analyses of PEG10 reveal new aspects of genomic organization, transcription and translation. PLoS One 5: e8686, 2010.

6. Okabe H, Satoh S, Furukawa Y, et al: Involvement of PEG10 in human hepatocellular carcinogenesis through interaction with SIAH1. Cancer Res 63: 3043-3048, 2003.

7. Kainz B, Shehata M, Bilban M, et al: Overexpression of the paternally expressed gene 10 (PEG10) from the imprinted locus on chromosome 7q21 in high-risk B-cell chronic lymphocytic leukemia. Int J Cancer 121: 1984-1993, 2007.

8. Liu DC, Yang ZL and Jiang S: Identification of PEG10 and TSG101 as carcinogenesis, progression, and poor-prognosis related biomarkers for gallbladder adenocarcinoma. Pathol Oncol Res 17: 859-866, 2011.

9. Ono R, Nakamura K, Inoue K, et al: Deletion of Peg10, an imprinted gene acquired from a retrotransposon, causes early embryonic lethality. Nat Genet 38: 101-106, 2006.

10. Smallwood A, Papageorghiou A, Nicolaides K, et al: Temporal regulation of the expression of syncytin $(H E R V-W)$, maternally imprinted $P E G 10$, and $S G C E$ in human placenta. Biol Reprod 69: 286-293, 2003.

11. Hu C, Xiong J, Zhang L, et al: PEG10 activation by co-stimulation of CXCR5 and CCR7 essentially contributes to resistance to apoptosis in $\mathrm{CD} 19^{+} \mathrm{CD} 34^{+} \mathrm{B}$ cells from patients with B cell lineage acute and chronic lymphocytic leukemia. Cell Mol Immunol 1: 280-294, 2004.

12. Chunsong H, Yuling H, Li W, et al: CXC chemokine ligand 13 and $\mathrm{CC}$ chemokine ligand 19 cooperatively render resistance to apoptosis in $\mathrm{B}$ cell lineage acute and chronic lymphocytic leukemia CD23 ${ }^{+} \mathrm{CD}^{+} \mathrm{B}$ cells. J Immunol 177: 6713-6722, 2006.

13. Xiong J, Qin J, Zheng Y, et al: PEG10 promotes the migration of human Burkitt's lymphoma cells by up-regulating the expression of matrix metalloproteinase-2 and -9. Clin Invest Med 35: E117-E125, 2012.

14. UyBico SJ, Wu CC, Suh RD, et al: Lung cancer staging essentials: the new TNM staging system and potential imaging pitfalls. Radiographics 30: 1163-1181, 2010.

15. Murchison JT: TNM staging update for lung cancer: why is this important? World J Radiol 4: 126-127, 2012.

16. Han R, Xiong J, Xiao R, et al: Activation of $\beta$-catenin signaling is critical for doxorubicin-induced epithelial-mesenchymal transition in BGC-823 gastric cancer cell line. Tumor Biol 34: 277-284, 2013.

17. Huang Q, Gumireddy K, Schrier M, et al: The microRNAs miR-373 and miR-520c promote tumour invasion and metastasis. Nat Cell Biol 10: 202-210, 2008.

18. Teng Y, Wang X, Wang Y and Ma D: Wnt/ $\beta$-catenin signaling regulates cancer stem cells in lung cancer A549 cells. Biochem Biophys Res Commun 392: 373-379, 2010.

19. Yang L, Chen Y, Cui T, et al: Desmoplakin acts as a tumor suppressor by inhibition of the Wnt/ $\beta$-catenin signaling pathway in human lung cancer. Carcinogenesis 33: 1863-1870, 2012.

20. Su K, Huang L, Li W, et al: TC-1 (c8orf4) enhances aggressive biologic behavior in lung cancer through the Wnt/ $\beta$-catenin pathway. J Surg Res 185: 255-263, 2013.

21. Thiery JP, Acloque H, Huang RY and Nieto MA: Epithelialmesenchymal transitions in development and disease. Cell 139: 871-890, 2009.

22. Acloque H,Adams MS,FishwickK, et al: Epithelial-mesenchymal transitions: the importance of changing cell state in development and disease. J Clin Invest 119: 1438-1449, 2009.

23. Nieto MA: Epithelial-mesenchymal transitions in development and disease: old views and new perspectives. Int J Dev Biol 53: 1541-1547, 2009. 
24. Shintani Y, Okimura A, Sato K, et al: Epithelial to mesenchymal transition is a determinant of sensitivity to chemoradiotherapy in non-small cell lung cancer. Ann Thorac Surg 92: 1794-1804, 2011.

25. Lee KR, Lee JS, Kim YR, et al: Polysaccharide from Inonotus obliquus inhibits migration and invasion in B16-F10 cells by suppressing MMP-2 and MMP-9 via downregulation of NF- $\kappa \mathrm{B}$ signaling pathway. Oncol Rep 31: 2447-2453, 2014.

26. Liu N, Li Y, Su S, et al: Inhibition of cell migration by ouabain in the A549 human lung cancer cell line. Oncol Lett 6: 475-479, 2013.

27. Jemal A, Bray F, Center MM, et al: Global cancer statistics. CA Cancer J Clin 61: 69-90, 2011.

28. Malvezzi M, Bertuccio P, Levi F, et al: European cancer mortality predictions for the year 2013. Ann Oncol 24: 792-800, 2013.

29. Goldstraw P, Crowley J, Chansky K, et al: The IASLC Lung Cancer Staging Project: proposals for the revision of the TNM stage groupings in the forthcoming (seventh) edition of the TNM Classification of Malignant Tumours. J Thorac Oncol 2: 706-714, 2007.

30. Siegel R, Naishadham D and Jemal A: Cancer statistics, 2012. CA Cancer J Clin 62: 10-29, 2012.
31. Heuberger $\mathbf{J}$ and Birchmeier W: Interplay of cadherin-mediated cell adhesion and canonical Wnt signaling. Cold Spring Harb Perspect Biol 2: a002915, 2010.

32. Johnson M, Sharma M, Jamieson C, et al: Regulation of $\beta$-catenin trafficking to the membrane in living cells. Cell Signal 21: 339-348, 2009.

33. Valenta T, Hausmann $\mathrm{G}$ and Basler $\mathrm{K}$ : The many faces and functions of $\beta$-catenin. EMBO J 31: 2714-2736, 2012.

34. Baum B, Settleman J and Quinlan MP: Transitions between epithelial and mesenchymal states in development and disease. Semin Cell Dev Biol 19: 294-308, 2008.

35. Thiery JP: Epithelial-mesenchymal transitions in development and pathologies. Curr Opin Cell Biol 15: 740-746, 2003.

36. Yang MH, Chen CL, Chau GY, et al: Comprehensive analysis of the independent effect of twist and snail in promoting metastasis of hepatocellular carcinoma. Hepatology 50: 1464-1474, 2009.

37. McDonnell S, Morgan M and Lynch C: Role of matrix metalloproteinases in normal and disease processes. Biochem Soc Trans 27: 734-740, 1999.

38. Shuman Moss LA, Jensen-Taubman S and Stetler-Stevenson WG: Matrix metalloproteinases: changing roles in tumor progression and metastasis. Am J Pathol 181: 1895-1899, 2012. 